\title{
Isolation and culture of primary osteocytes from the long bones of skeletally mature and aged mice
}

Amber Rath Stern ${ }^{1, *}$, Matthew M. Stern ${ }^{1, *}$, Mark E. Van Dyke ${ }^{2}$, Katharina Jähn ${ }^{1}$, Matthew Prideaux ${ }^{1}$, and Lynda F. Bonewald ${ }^{1}$

${ }^{1}$ University of Missouri Kansas City, Kansas City, MO, USA and ${ }^{2}$ Wake Forest Institute for Regenerative Medicine, Winston Salem, NC, USA

${ }^{*}$ A.R.S and M.M.S. contributed equally to this work.

BioTechniques 52:361-373 (June 2012) doi 10.2144/0000113876

Keywords: osteocyte; isolation; age; culture; collagenase; mice

Supplementary material for this article is available at www.BioTechniques.com/article/113876

The purpose of this work was to establish a methodology to enable the isolation and study of osteocytes from skeletally mature young (4-month-old) and old (22-month-old) mice. The location of osteocytes deep within bone is ideal for their function as mechanosensors. However, this location makes the observation and study of osteocytes in vivo technically difficult. Osteocytes were isolated from murine long bones through a process of extended collagenase digestions combined with EDTA-based decalcification. A tissue homogenizer was used to reduce the remaining bone fragments to a suspension of bone particles, which were placed in culture to yield an outgrowth of osteocyte-like cells. All of the cells obtained from this outgrowth that displayed an osteocyte-like morphology stained positive for the osteocyte marker E11/GP38. The osteocyte phenotype was further confirmed by a lack of staining for alkaline phosphatase and the absence of collagen la 1 expression. The outgrowth of osteocytes also expressed additional osteocyte-specific genes such as Sost and Mepe. This technique facilitates the isolation of osteocytes from skeletally mature bone. This novel enabling methodology should prove useful in advancing our understanding of the roles mature osteocytes play in bone health and disease.

The coordinated actions of three different types of bone cells are required for bone remodeling in response to mechanical loading. On the surface of bone are osteoblasts, which form new bone, and osteoclasts, which remove bone. Located deeper within the bone matrix and housed in cave-like lacunae, are osteocytes, which function as the mechanosensory system within bone. Osteocytes have a dendritic morphology with many cell processes extending through canaliculi to form a highly connected communication network between themselves and the bone surface cells. When mechanical loads are applied to bone, they are sensed by osteocytes, which translate signals provided by mechanostimulation into biochemical signals. These signals are believed to regulate the actions of osteoblasts and osteoclasts, thereby providing a mechanism to regulate bone deposition and absorption according to the local mechanical requirements of the bone (1-3).

Osteocytes are the most abundant of the three bone cell types; however, the least is known about them. While their location deep within the bone matrix makes them ideally situated to sense bone strain, it also makes their observation and study in vivo difficult. Additionally, primary osteocytes, particularly those within the long bones of skeletally mature animals, have proven difficult to obtain and study ex vivo. Furthermore, once primary osteocytes are obtained, their study is often limited by their inability to proliferate as they are considered terminally differentiated cells.

To facilitate the study of osteocytes in vitro, Kato et al. created the immortalized osteocyte-like MLO-Y4 line from osteocytes isolated from the long bones of 6-week-old transgenic mice, in which the SV40 large $\mathrm{T}$-antigen oncogene is expressed under the control of an osteocalcin promoter $(4,5)$. The MLO-Y4 cell line is well-characterized and represents the phenotype of early osteocytes $(4,6)$. This osteocyte-like cell line has been used to study communication between osteocytes, as well as communication between osteocytes and bone surface cells (7-13). MLO-Y4 cells have also been used in the investigation of the response of osteocytes to various types of physical stimulation, including fluid flow and mechanical perturbations (7-20). Although the MLO-Y4 cell line is a very powerful tool for the study of osteocytes in vitro, there are known differences between primary osteocytes and the immortalized MLO-Y4 cell line. For example, MLO-Y4 cells express low to undetectable levels of Dentin matrix protein 1 (Dmpl) and Sclerostin (Sost), while osteocytes are known to express these genes in vivo (6,21-29). The MLO-A5 cell line has characteristics of a postosteoblast/preosteocyte. These cells are very large, over $100 \mathrm{~nm}$, express all of the markers of the late osteoblast such as extremely high alkaline phosphatase (ALP), bone sialoprotein, PTH type 1 receptor, and osteocalcin, and rapidly mineralize in sheets, not nodules (30). In culture, these cells begin to express markers of osteocytes as they generate cell processes such as E11 (31), but express low levels of Sost. 

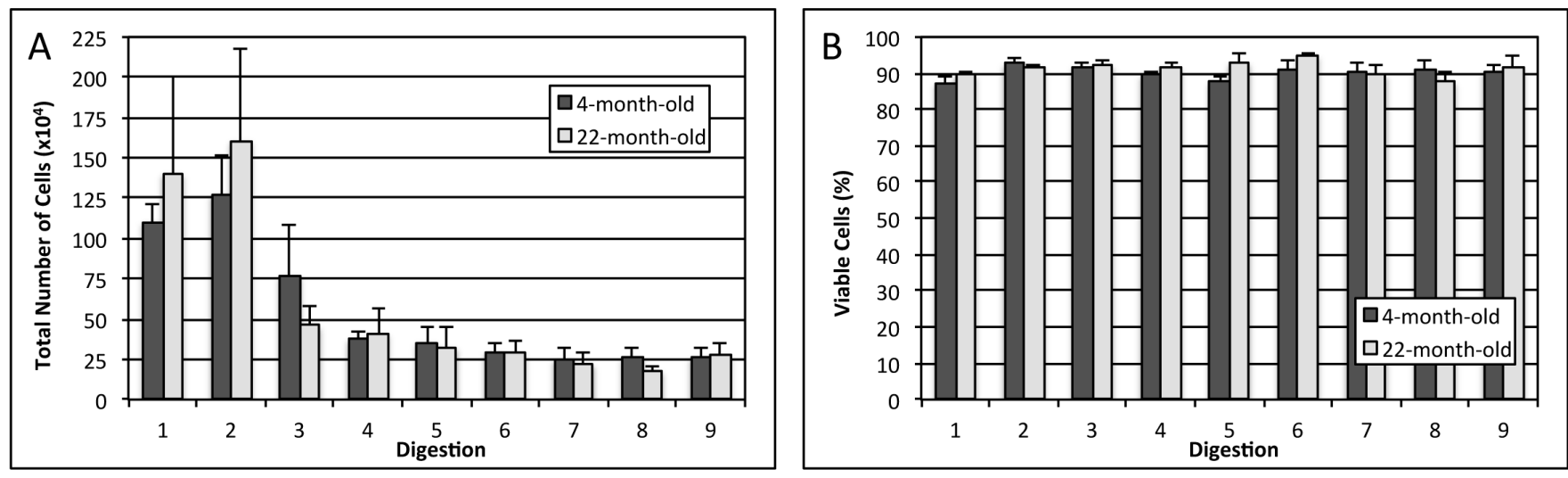

Figure 1. Total number of cells and percentage of viable cells obtained at each stage of digestion during osteocyte isolation. (A) Total number of cells obtained from the combined long bones of a single mouse during each stage of digestion from the long bones of a single mouse. (B) The percentage of viable cells isolated during each stage of digestion based on trypan blue staining ( $n=4$ for both the 4-month-old and 22-month-old mice).

Recently, a cell line has been generated that differentiates from the late osteoblast to the late osteocyte called IDG-SW3, made by crossing the Immortomouse with the 8-kb Dmpl-GFP transgenic mouse line. IDG-SW3 cells synthesize and mineralize a "honeycomb-like" matrix rich in type-I collagen similar to MLO-A5 cells. Similar to osteocytes in vivo, these cells express osteocyte marker genes from the early osteocyte marker E11 to the mature osteocyte marker sclerostin, down-regulate Sost expression with PTH treatment and increase Fgf 23 mRNA expression in response 1,25-dihydroxyvitamin $\mathrm{D}_{3}$ (32). This cell line faithfully recapitulates the differentiation process from osteoblast to late osteocyte as observed in vivo.

While these cell lines are convenient and useful, an ideal strategy for the study of osteocyte function ex vivo, and validation of results obtained with cell lines is to develop better methodology for the isolation and study of primary osteocytes.

Primary osteocytes have most commonly been isolated from 16- or 18-day-old chick calvaria $(15,33-44)$ or from newborn through 4-day-old rat calvaria, 12-day-old mouse calvaria (45), and 3- to 4-week-old mouse calvaria and long bones (46). More recently, osteocytes have also been isolated from the long bones of 3-day-old SpragueDawley rats (33). Calvaria from young chicks and neonatal rats and mice, as well as the long bones from neonatal rats and young mice (3-4 weeks) are all very thin and easily processed using sequential digestions with EDTA and collagenase. Studies utilizing these primary osteocytes can provide insight to the behavior of osteocytes during development but do not aid in the study of osteocytes from skeletally mature animals or enable the comparison between osteocytes isolated from skeletally mature but relatively
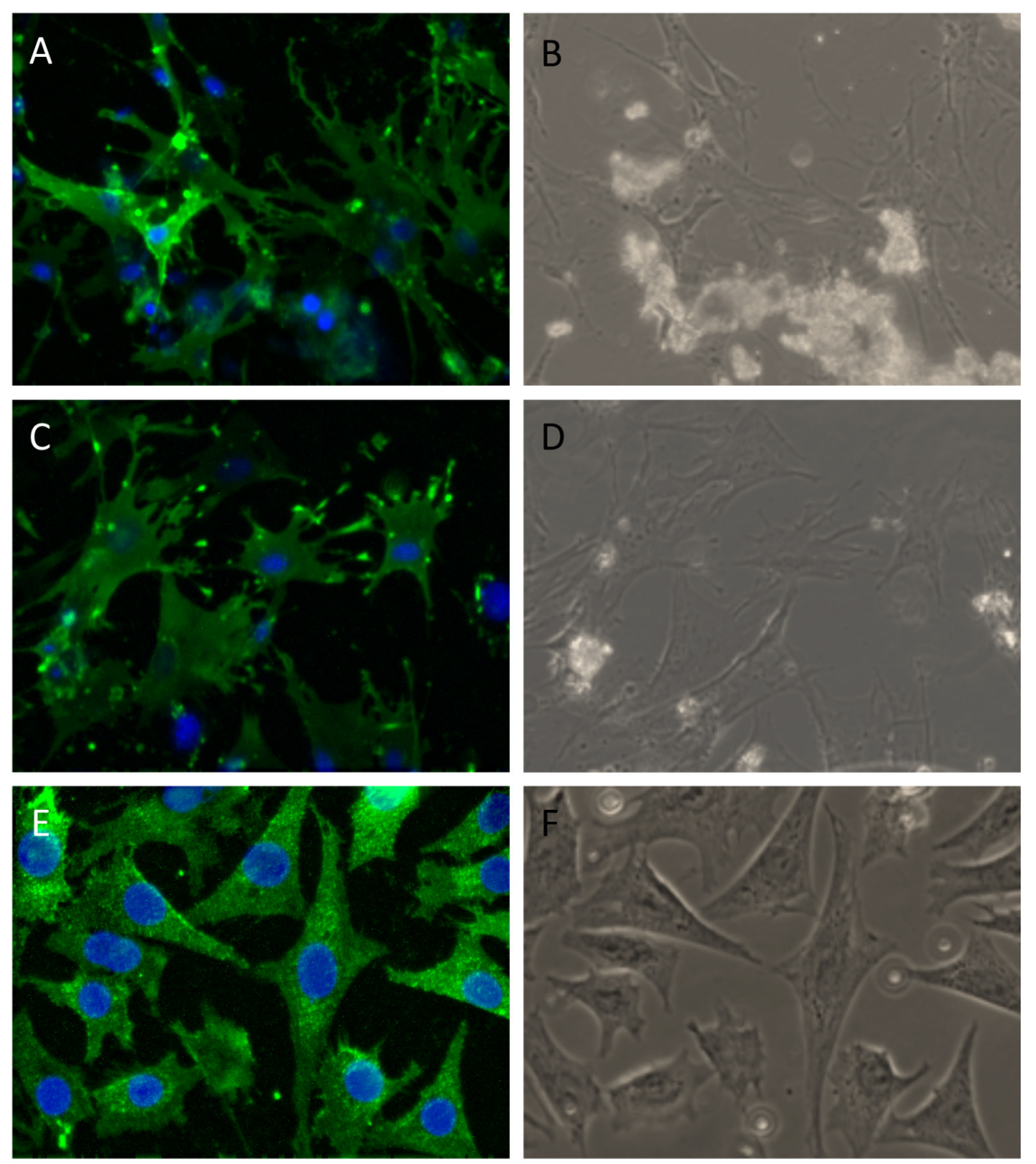

Figure 2. Primary osteocytes display an osteocyte-like morphology and express E11/GP38. After 7 days in culture, a majority of the ( $A$ and $B$ ) young and $(C$ and $D)$ old cells from the bone particle outgrowth displaying an osteocyte-like morphology also stained positive for E11/GP38 (green). (E and F) MLO-Y4 osteocyte-like cells were used as a positive control for the E11/GP38 staining. DAPI (blue) stain was utilized to identify cell nuclei and count cells. Phase-contrast images of the same fields $(C$ and $D$ ) are shown for comparison (magnification $=200 \times$ in all images). 
Table 1. Osteocyte isolation from murine long bone

\begin{tabular}{|c|c|}
\hline Step & Description \\
\hline 1. & Murine long bones (femurs, tibiae, and humeri) were dissected. \\
\hline 2. & Bones were placed in $\alpha \mathrm{MEM}$ with $10 \%$ penicillin and streptomycin. \\
\hline 3. & $\begin{array}{l}\text { Soft tissue (any remaining muscle and connective tissue) and periosteum were removed } \\
\text { through scraping and extensive washing. }\end{array}$ \\
\hline 4. & Bones were placed in fresh $\alpha$ MEM with $10 \%$ penicillin and streptomycin. \\
\hline 5. & $\begin{array}{l}\text { Epiphyses were cut off, and the marrow was flushed out with HBSS using a syringe or by } \\
\text { centrifugation. }\end{array}$ \\
\hline 6. & Bones were washed, cut lengthwise, and then cut into 1- to 2-mm lengths. \\
\hline 7. & Bone pieces were placed in HBSS. \\
\hline 8. & $\begin{array}{l}\text { Bone pieces were incubated in collagenase solution for } 25 \mathrm{~min} \text {, the solution was aspirated } \\
\text { and kept for cell plating, and the bone pieces were washed in HBSS. This was repeated two } \\
\text { more times, for a total of three digestions. }\end{array}$ \\
\hline 9. & $\begin{array}{l}\text { Bone pieces were incubated with EDTA solution for } 25 \text { min, the solution was aspirated and } \\
\text { kept for cell plating, and the bone pieces were washed in HBSS (digestion 4). }\end{array}$ \\
\hline 10. & $\begin{array}{l}\text { Bone pieces were incubated with collagenase solution for } 25 \text { min, the solution was aspirated } \\
\text { and kept for cell plating, and the bone pieces were washed in HBSS (digestion 5). }\end{array}$ \\
\hline 11. & $\begin{array}{l}\text { Bone pieces were incubated with EDTA solution for } 25 \text { min, the solution was aspirated and } \\
\text { kept for cell plating, and the bone pieces were washed in HBSS (digestion 6). }\end{array}$ \\
\hline 12. & $\begin{array}{l}\text { Bone pieces were incubated with collagenase solution for } 25 \mathrm{~min} \text {, the solution was aspirated } \\
\text { and kept for cell plating, and the bone pieces were washed in HBSS (digestion 7). }\end{array}$ \\
\hline 13. & $\begin{array}{l}\text { Bone pieces were incubated with EDTA solution for } 25 \text { min, the solution was aspirated and } \\
\text { kept for cell plating, and the bone pieces were washed in HBSS (digestion 8). }\end{array}$ \\
\hline 14. & $\begin{array}{l}\text { Bone pieces were incubated with collagenase solution for } 25 \mathrm{~min} \text {, the solution was aspirated } \\
\text { and kept for cell plating, and the bone pieces were washed in HBSS (digestion 9). }\end{array}$ \\
\hline 15. & $\begin{array}{l}\text { Remaining bone pieces were minced in } \alpha M E M \text { utilizing a tissue homogenizer and the } \\
\text { resulting suspension of bone particles was directly plated. }\end{array}$ \\
\hline
\end{tabular}

Table 2. RT-PCR primers used.

\begin{tabular}{|c|c|c|c|}
\hline Gene & Gene symbol & $\begin{array}{l}\text { TaqMan gene } \\
\text { expression assay primer }\end{array}$ & $\begin{array}{l}\text { Amplicon } \\
\text { length (bp) }\end{array}$ \\
\hline Podoplanin & $\begin{array}{l}\text { E11/Pdpn/ } \\
\text { Gp38 }\end{array}$ & Mm00494716_m1 & 86 \\
\hline Sclerostin & Sost & Mm00470479_m1 & 55 \\
\hline Fibroblast growth factor 23 & Fgf23 & Mm00445621_m1 & 98 \\
\hline Prostaglandin-endoperoxide synthase 2 & $\operatorname{Cox} 2$ & Mm01307329_m1 & 97 \\
\hline $\begin{array}{l}\text { Matrix extracellular phosphoglycoprotein } \\
\text { with ASARM motif }\end{array}$ & Mepe & Mm02525159_s1 & 152 \\
\hline $\begin{array}{c}\text { Phosphate regulating gene with } \\
\text { homologies to endopeptidases on the } \mathrm{X} \\
\text { chromosome }\end{array}$ & Phex & Mm00448119_m1 & 137 \\
\hline Dentin matrix protein 1 & Dmp1 & Mm00803831_m1 & 91 \\
\hline$\beta-2$ microglobulin & $\beta 2 m$ & Mm00437762_m1 & 77 \\
\hline
\end{tabular}

young mice (4- to 6-month) and aged mice (>22-month-old).

The purpose of the work presented here was to create an osteocyte isolation technique to enable the isolation and study of osteocytes from the long bones of young mature and old adult mice.

\section{Materials and methods}

\section{Primary osteocyte isolation}

Osteocytes were isolated from mouse long bones utilizing a modified protocol derived from the combined methods of $\mathrm{Gu}$ et al. and Van Der Plas et al. $(33,44,47)$. Long bones (femora, tibia, and humeri) were aseptically dissected from skeletally mature 4-month-old (young) and 22-month-old (old) C57BL/6 mice (Charles River Laboratories, Wilmington, MA, USA). The bones from young and old mice were processed separately by serial digestion as described in Table 1 (see Supplementary material for a detailed protocol). The bones from each individual mouse were pooled together and treated as one sample. Collagenase solution was prepared as 300 active $\mathrm{U} / \mathrm{mL}$ collagenase type-IA (Sigma-Aldrich, St. Louis, MO, USA) dissolved in $\alpha$-minimal essential medium ( $\alpha$ MEM; Mediatech, Manassas, VA, USA).EDTA tetrasodium salt dehydrate (EDTA) solution ( $5 \mathrm{mM}$, pH = 7.4; Sigma-
Aldrich) was prepared in magnesium and calcium-free Dulbecco's phosphate-buffered solution (DPBS; Mediatech) with 1\% BSA (Sigma-Aldrich). All steps of the digestion took place in $8 \mathrm{~mL}$ solution in a six-well Petri dish, on a rotating shaker set to $200 \mathrm{RPM}$, in a $37^{\circ} \mathrm{C}$ and $5 \% \mathrm{CO}_{2}$ humidified incubator. Following each sequential digestion, the digest solution with suspended cells was removed from the bone pieces and kept. The bone pieces were then rinsed with Hank's balanced salt solution (HBSS) three times, and the rinsate was added to the digestion solution. The combined cell suspension solution was spun down at $200 \times g$ for $5 \mathrm{~min}$, the supernatant was removed from the cell pellet, and cells were resuspended in culture medium and counted. The tissue homogenizer used in this study (Medimachine; BD Biosciences, San Jose, CA, USA) was utilized with a stainless steel mincing screen with a pore size of $50 \mu \mathrm{m}$.

Primary bone cell culture

Cell suspensions resulting from the primary isolation procedure (detailed in section above) were cultured on type-I rat tail collagencoated six-well plates (Becton Dickinson, Franklin Lakes, NJ, USA) at a seeding density of approximately 250,000 cells per $9.5 \mathrm{~cm}^{2}$ in $\alpha$-minimal essential medium ( $\alpha$ MEM; Mediatech) supplemented with 5\% fetal bovine serum (FBS; Summit Biotechnology, Fort Collins, CO, USA), $5 \%$ calf serum (CS; HyClone Laboratories, Logan, UT, USA), and $1 \%$ penicillin and streptomycin (PS; Cellgro, Manassas, VA, USA). Cells were maintained at $37^{\circ} \mathrm{C}$ and $5 \% \mathrm{CO}_{2}$ in a humidified incubator for 7 days.

\section{Culture of MLO-Y4 cells}

MLO-Y4 cells, a murine osteocyte-like immortalized cell line, were utilized as control cells in these experiments $(4,5)$. They were cultured on type-I rat tail collagen-coated tissue culture plates in $\alpha$-MEM supplemented with $2.5 \% \mathrm{FBS}, 2.5 \% \mathrm{CS}$, and $1 \% \mathrm{PS}$. The cells were maintained in a $37^{\circ} \mathrm{C}$ and $5 \%$ $\mathrm{CO}_{2}$ humidified incubator and subcultured approximately every $72 \mathrm{~h}$ to maintain the cell populations at less than $70 \%$ confluent.

Culture and differentiation of

MC3T3-E1 cells

The MC3T3-E1 cell line (subclone 4; ATCC, Manassas, VA, USA) was also utilized as control cells in these experiments. They exhibit markers of osteoblast differentiation, particularly expression of ALP after growth in ascorbic acid and phosphate, but prior to differentiation do not express ALP. MC3T3-E1 cells were cultured on type-I rat tail collagen-coated tissue culture plates in $\alpha$-MEM, supplemented with $10 \%$ 

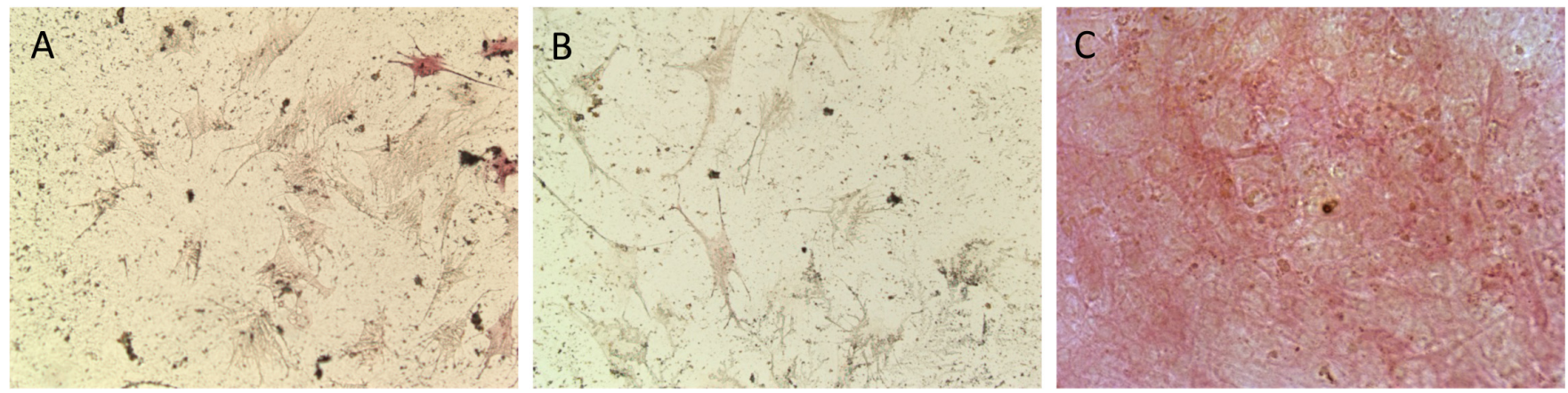

Figure 3. ALP staining. ALP staining of cells isolated from (A) young (4-month-old) and (B) old (22-month-old) mouse bone particles. (C) MC3T3-E1 cells differentiated along the osteogenic lineage were utilized as positive controls.
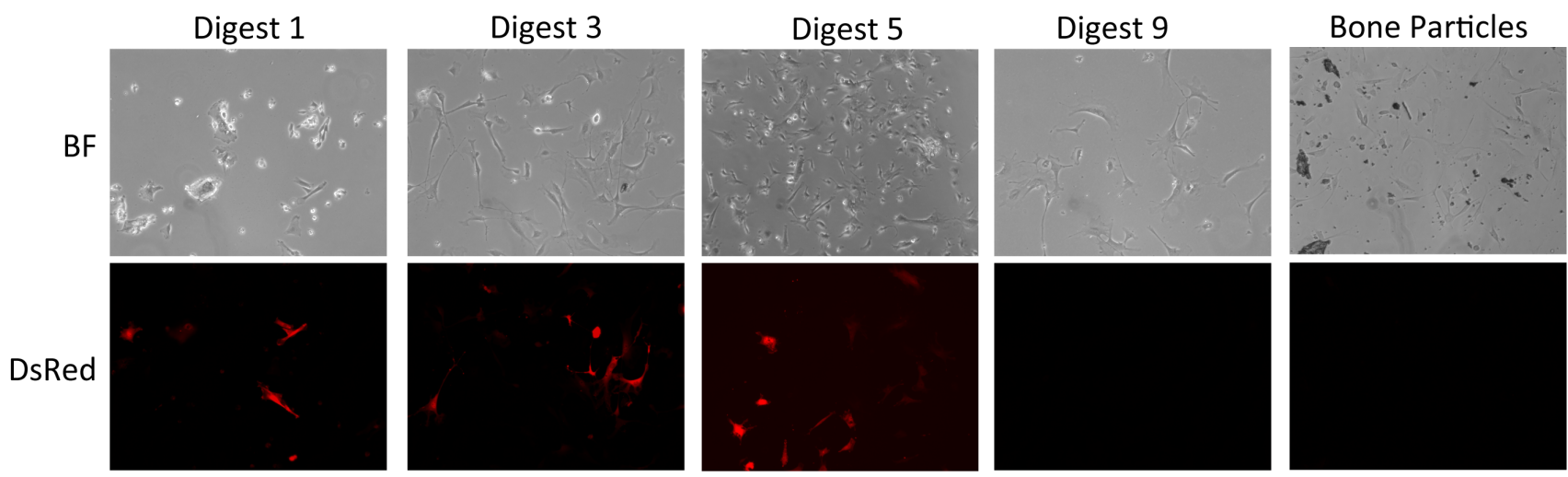

Figure 4. DsRed expression in cells obtained during the osteocyte isolation procedure carried out on COL1a1-DsRed transgenic mice. Bright field (BF) and fluorescent images were captured from five random fields of each of the cell populations isolated from each digestion. Representative images from the odd digestions are included here.

FBS, 1\% PS. To induce osteogenic differentiation, $50 \mu \mathrm{g} / \mathrm{mL}$ ascorbic acid and $10 \mathrm{mM}$ $\beta$-glycerophosphate were added to the culture media. The cells were allowed to differentiate for 14 days, changing the media as needed.

\section{Culture of SW 3 cells}

The IDG-SW3 cell line were utilized as control cells for investigating osteocyte marker expression as these cells have previously been shown to express mature osteocyte markers, such as Mepe, Sost, and Fgf23, when differentiated (32). Cells were cultured in $\alpha$-MEM, supplemented with $10 \% \mathrm{FBS}, 1 \% \mathrm{PS}$, and $50 \mathrm{U} / \mathrm{mL}$ interferon- $\gamma$ (IFN- $\gamma$; Invitrogen, Carlsbad, CA, USA), to induce proliferation. Upon reaching confluence, the media was replaced with differentiation media, containing $\alpha$-MEM, $10 \%$ FBS, $1 \%$ PS, $50 \mu \mathrm{g} / \mathrm{mL}$ ascorbic acid, and $4 \mathrm{mMB}$-glycerophosphate, to induce osteogenic differentiation. The media was replaced every 3 days, and the cells were differentiated for 21 days.

Viable cell counts

Following each digestion, cells were counted using a hemocytometer before being plated. A trypan blue (MP Biomedicals, Solon,

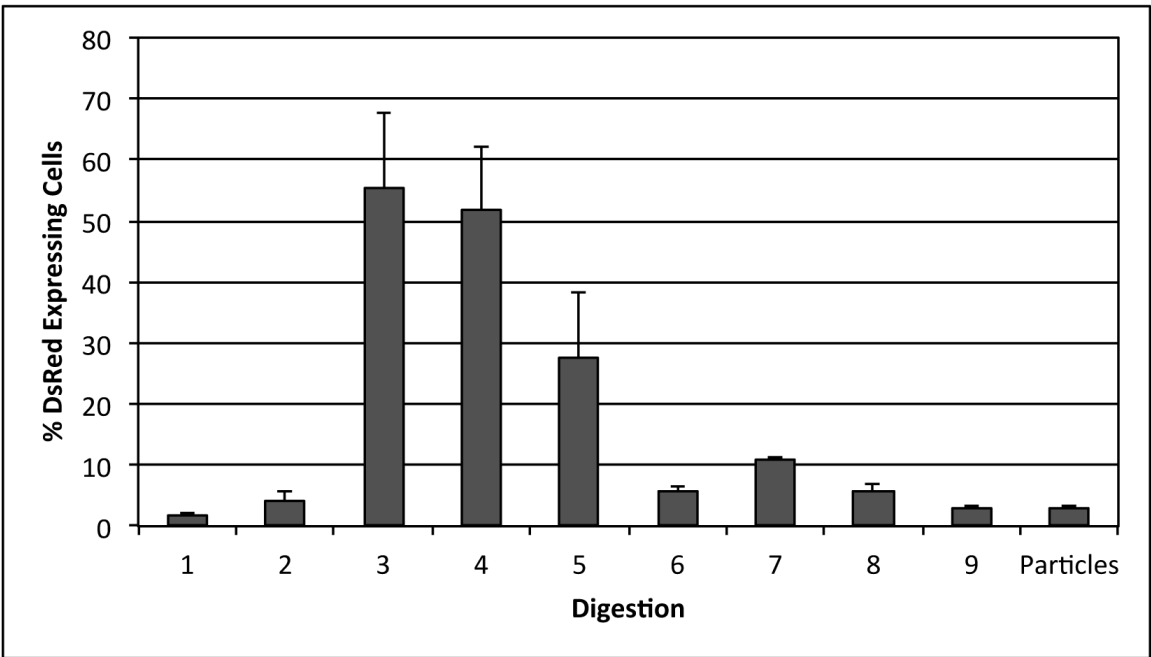

Figure 5. COL1A1 expression in cells obtained at each stage of the osteocyte isolation procedure. The percentage of cells expressing DsRed, which was driven by the COL1A1 promoter, was calculated for each digestion 7 days post-isolation $(n=2)$.

$\mathrm{OH}, \mathrm{USA}$ ) exclusion test for cell viability was utilized to determine the number of dead cells in each of the cell isolations. The percentage of viable cells was calculated for each digestion for cell isolations from both the young and old bones.
Immunostaining for E11/gp38

Primary osteocytes isolated from 4- and 22 -month-old mice were immunologically identified using an anti-E11/GP38 antibody 7 days following the isolation procedure. In osteocytes, E11 has been 

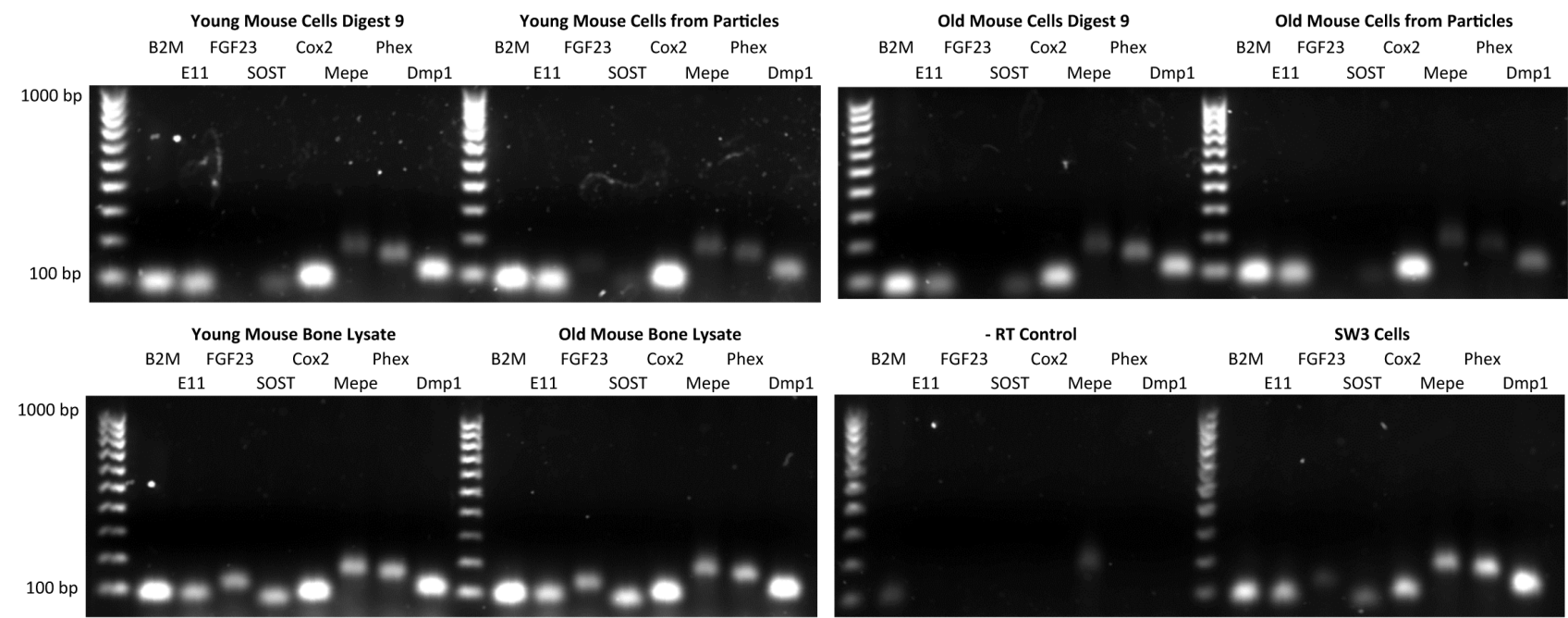

Figure 6. Gene expression of the cells obtained during the osteocyte isolation procedure. RT-PCR was used to assess the expression of genes characteristic of osteocytes with total bone lysate and SW3 cells used as positive controls. $\beta 2 m$ was utilized as a housekeeping gene, and -RT reactions confirmed that genomic DNA was not present. All products were of the expected size; 100-bp ladder.

shown to be responsible for dendrite elongation and is a marker of early osteocytes. The bone cells isolated from the young and old bone particles at 7 days post-isolation, and control MLO-Y4 cells were fixed with $4 \%$ paraformaldehyde in PBS for $10 \mathrm{~min}$, then incubated with PBS for $2 \mathrm{~min}$. The cells were then incubated for $45 \mathrm{~min}$ at $25^{\circ} \mathrm{C}$ with gentle shaking with blocking solution: PBS $+10 \%$ goat serum (HyClone Laboratories). Primary antibody, podoplanin (8.1.1; Santa Cruz Biotechnology, Santa Cruz, CA, USA) at a 1:50 dilution, was applied in PBS + 3\% goat serum overnight at $4^{\circ} \mathrm{C}$ with shaking. After washing with PBS, the secondary antibody-Alexa Fluor 488-labeled goat anti-hamster IgG (Molecular Probes, Eugene, OR, USA) at $10 \mu \mathrm{g} / \mathrm{mL}$ was applied for $45 \mathrm{~min}$ at $25^{\circ} \mathrm{C}$ with gentle shaking. Cells were then washed with PBS, counterstained with 4',6-diamidino-2phenylindole (DAPI; Vector, Burlingame, CA, USA), and mounted prior to imaging by fluorescence microscopy. The percentage of osteocytes within the cell isolates was quantified by determining the percentage of E11/GP38+ cells present using DAPI to quantify the total number of cells. A total of 10 random fields of each isolate were counted at $200 \times$ magnification.

\section{ALP staining}

ALP staining was conducted on the cell populations isolated from the out growth of cells from the bone particles for young (4-month-old) and old (22-month-old) murine long bones 7 days post-isolation. The media was removed and the cells washed with DPBS, fixed with $4 \%$ paraformaldehyde for 2 min, rinsed with DPBS, and then incubated at ambient temperature on a shaker with ALP staining solution for $30 \mathrm{~min}$. A 25 - $\mathrm{mL}$ formulation of the staining solution consisted of 24 $\mathrm{mL}$ MilliQ water, $1 \mathrm{~mL}$ Napthol AS-MX phosphate alkaline solution-0.25\% (SigmaAldrich), and $25 \mathrm{mg}$ Fast Violet B salts (Sigma-Aldrich). The ALP staining solution was removed and cells were coverslipped. For each cell population, 10 random fields were unique in vivo siRNA delivery reagents $\underset{\text { from }}{\text { fapan }}$

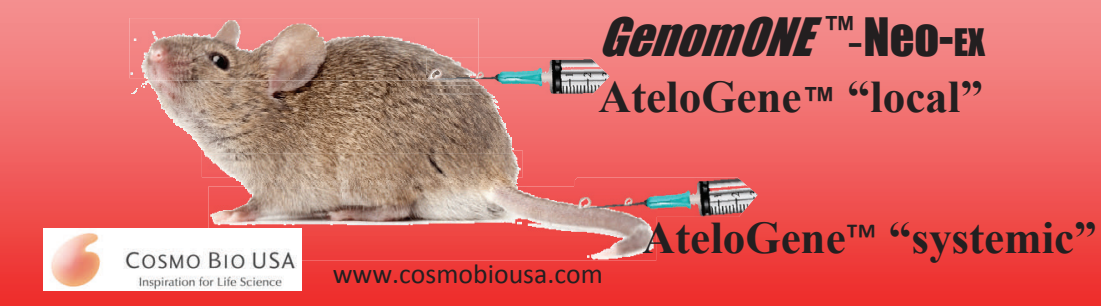

imaged at $200 \times$ magnification. Fibroblast-like (ALP-negative elongated spindle-shaped cells), osteoblast-like (ALP-positive and cuboidal in shape), and osteocyte-like (ALP-negative or only weakly positive stellate cells) cell fractions were quantified for the osteocyteenriched cell populations isolated from the final bone particle digestion for the bones isolated from young (4-month-old) and old (22-month-old) mice. MLO-Y4 cells, undifferentiated MC3T3-E1 cells, and differentiated MC3T3-E1 cells were also stained at the same time and used as controls.

\section{Collal DsRed mouse cell isolation}

The osteocyte isolation procedure was utilized to procure cells from collagen DsRed mice (generously provided by Dr. David Rowe, University of Connecticut Health Center) ( $n=2$, age $=4$ months). These mice express the DsRed reporter gene under control of the murine 3.6-kb Collal promoter. Cells expressing COL1A1, a marker of osteoblasts, can be positively identified by their DsRed reporter activity and distinguished from mature osteocytes, which are negative for COL1A1 expression. Seven days postisolation, the cell populations from the final digestion and those from the bone particle outgrowth were analyzed. Bright field and fluorescent images were captured at 100X magnification. A total of five fields were randomly imaged per digestion, and the percentage of DsRed-positive cells was calculated. Cells isolated from the early digests were used as a positive control, as these are the osteoblast-enriched digestions (33).

Gene expression

Seven days post-isolation, RNA was isolated from the outgrowth of cells from the fine 
bone particle digestion as well as the plated cells from digest 9 in both the young and old isolations using the RNeasy Plus mini kit (Qiagen,Valencia, CA, USA) and reversetranscribed using the SuperScript III FirstStrand Synthesis SuperMix for qRT-PCR (Invitrogen). Reactions lacking RT, bonelysate from both young and old bones, and SW 3 cells were utilized as controls. The bone lysate was derived from the bone particles obtained from the tissue homogenizer in the final stage of the cell isolation process. RT-PCR was performed for the following osteocytic genes: E11, Sost, Fgf23, Cox2, Mepe, Phex, and Dmp1 using commercially available primers (Table 2 ). $\beta 2 m$ was used as the endogenous control. RT-PCR products were visualized on a $1 \%$ agarose gel stained with ethydium bromide with a 100-1000 base pair ladder used for reference (Bio-Rad Laboratories, Hercules, CA, USA).

\section{Results and discussion}

The isolation procedure (described in primary osteocyte isolation section of Materials and methods) results in a highly osteocyte-enriched population of primary cells within the final digestion as well as from the outgrowth of additional cells from the bone particles obtained during processing in the tissue homogenizer. The isolated cells exhibited the classic osteocyte morphology with long dendritic cell processes projecting away from the cell body and had an osteocyte-like phenotype.

\section{Viable cell counts}

To determine how many cells could be obtained per mouse and if the cells survived the isolation procedure, the number and viability of the cells was examined following each stage of the process. There was a large variation in the number of cells isolated during the initial digestions, but the later digestions consistently produced around 250,000 cells per digestion per mouse (Figure 1). Approximately $90 \%$ of the cells in each digestion were viable based on trypan blue staining.

Table 3. Quantification of cell types in late digestions based on ALP expression and morphology.

\begin{tabular}{|c|c|c|c|}
\hline \multicolumn{4}{|c|}{ Murine long bone osteocyte enriched digestions } \\
\hline Cell population & $\begin{array}{c}\text { Fibroblast-like cells }^{\mathbf{a}} \\
\mathbf{( \% )}\end{array}$ & $\begin{array}{c}\text { Osteoblast-like cells } \\
(\mathbf{\%})\end{array}$ & $\begin{array}{c}\text { Osteocyte-like } \text { cells }^{\mathbf{c}} \\
(\%)\end{array}$ \\
\hline Digestion 6 & 20.93 & 48.84 & 30.23 \\
\hline Digestion 7 & 11.11 & 25.93 & 62.96 \\
\hline Digestion 8 & 5.41 & 31.08 & 63.51 \\
\hline Digestion 9 & 10.64 & 19.15 & 70.21 \\
\hline Young bone particles & 4.49 & 15.73 & 79.78 \\
\hline Old bone particles & 5.36 & 9.82 & 84.82 \\
\hline
\end{tabular}

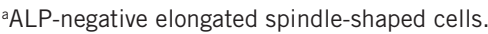

${ }^{\mathrm{b}} \mathrm{ALP}$-positive and cuboidal in shape.

ALP-negative (or only weakly positive) stellate cells.

\begin{tabular}{|c|c|c|c|}
\hline \multicolumn{4}{|c|}{ ALP staining controls } \\
\hline Cell population & $\begin{array}{c}\text { ALP positive } \\
\mathbf{( \% )}\end{array}$ & $\begin{array}{c}\text { ALP faintly positive } \\
\mathbf{( \% )}\end{array}$ & $\begin{array}{c}\text { ALP negative } \\
\text { (\%) }\end{array}$ \\
\hline MLO-Y4 & 8.21 & 17.47 & 74.32 \\
\hline MC3T3-E1 & 0.00 & 0.00 & 100.00 \\
\hline MC3T3-E1 Differentiated & 99.50 & 0.50 & 0.00 \\
\hline
\end{tabular}

Fibroblast-like (ALP-negative elongated spindle-shaped cells), osteoblast-like (ALP-positive and cuboidal in shape), and osteocyte-like [ALP-negative (or only weakly positive) stellate cells] cell fractions were quantified for each of the osteocyte enriched digestion phases as well as the final bone particle digestion for the bones isolated from young (4-month-old) and old (22-month-old) mice. MLO-Y4 cells, undifferentiated MC3T3-E1 cells, and differentiated MC3T3-E1 cells expressing osteoblast markers were used as controls.

\section{E11/GP38 staining}

E11/GP38 is the earliest osteocyte-specific protein to be expressed as the osteoblast differentiates into an osteocyte (9). To determine what percentage of cells obtained during the final stage of the isolation procedure expressed E11/GP38, immunofluorescent staining was used. After 7 days in culture, the outgrowth of cells from the final bone particles (after all nine stages of digestion and processing in tissue homogenizer) of young (4-month-old) and old (22-month-old) murine long bones were fixed, immunofluorescently stained, and imaged. In the outgrowth of cells from both the young and old bone particles, $100 \%$ of the isolated cells that displayed an osteocyte-like morphology also stained positive for E11/

\section{Purifying $\lg G$ ?}

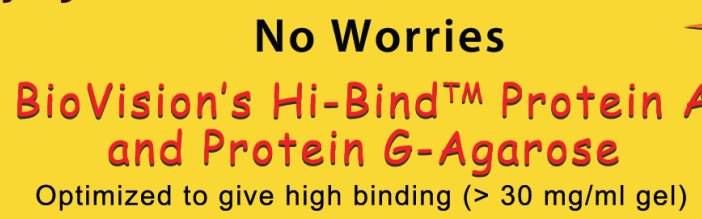

www.biovision.com

$ه 1.800 .891 .9699$

Vol. $52 \mid$ No. $6 \mid 2012$
GP38, thereby confirming the osteocyte phenotype (Figure 2). E11/GP38 is also highly expressed in MLO-Y4 osteocyte-like cells, which were used as a positive control for E11/ GP38 staining (6). A negative control further confirmed the specificity of the antibody (data not shown). Cells from the early stages of the digestions (digests 4 and 5) were also plated and stained. Approximately 50\% of these cells stained positive for E11/GP38 (data not shown).

\section{ALP staining}

ALP expression and activity is an indicator of the osteoblastic state and its expression is shut down in osteocytes. To determine what percentage of cells obtained during each stage of the isolation procedure express ALP, a colorimetric assay for ALP expression was performed. The percentage of osteocyte-like cells obtained during each of the digests, based on the lack of ALP staining and a stellate/dendritic morphology, increased with each sequential digestion (Table 3). The highest percentage of osteocyte-like cells was found in the outgrowth of cells from the bone particles that remained after the final digestion and processing in the tissue homogenizer (Figure 3).

COL1A1 DsRed mouse cell isolation Collagen type-I is a major component of bone and produced by osteoblasts. To determine if cells obtained during the isolation procedure 
were collagen I-producing osteoblasts, the osteocyte isolation was carried out on the bones of mice carrying a DsRed reporter gene under control of the murine 3.6-kb Collal promoter, and the percentage of DsRed positive cells obtained at each stage of the procedure was determined via fluorescent microscopy. After 7 days in culture, a total of five fields were randomly imaged per stage of digestion resulting in an average of 250 cells per digestion (Figure 4). The percent of cells expressing DsRed was highest in the cells obtained in digests 3-5 and decreased in the later digestions (Figure 5), suggesting that early and middle fractions were the most enriched for osteoblasts, while late fractions contained relatively few osteoblasts.

\section{Gene expression}

Osteocytes are known to express several genes that are both important for their function and can distinguish them from other bone cells. Osteoid osteocytes are known to express E11, Phex, and Mepe, while mineralizing osteocytes express Dmpl, and mature osteocytes encased in a mineralized matrix express Sost and Fgf23 (1). In osteocytes, COX2 is known to play a role in the regulation PGE2 (prostaglandin E2) production (6). To determine if cells isolated using the methodology described above possess a gene expression profile characteristic of osteocytes, RT-PCR was performed. In the harvest of bone cells from both young and old mice, cells within digest 9, as well as the outgrowth of cells from homogenized bone particles, expressed several genes characteristic of osteocytes, including: E11, Sost, Cox2, Mepe, Phex, and Dmp1 (Figure 6). Total bone lysate and the SW3 cell line (32) were used as positive controls. As can be seen, all genes were expressed to various levels in young mouse bone lysate, old mouse bone lysate, and SW3 cells. The young mouse digest 9 gene expression profile was very similar to the young mouse cells from particles. However, there was less Fgf 23 in digest 9 compared with the cells from the particles. The old mouse digest 9 was very similar to old mouse cells from particles where no Fgf 23 was detected. Although there appeared to be lower expression of Phex in the old mouse cells from particles, these experiments would have to be performed in triplicate to determine if significant differences exist.

In this study, we were able to successfully isolate primary cells displaying several characteristics of osteocytes from the long bones of skeletally mature 4-month-old and 22-month-old mice through a process of sequential digestions and the use of a tissue homogenizer. From both the 4-month-old and 22-month-old mice, approximately 250,000 cells per osteocyte-enriched digestion (digestions 7-9) were obtained. These cells expressed E11/GP38 protein, and they lacked ALP and
COL1A1 expression found in osteoblasts. Furthermore, several genes known to be expressed in osteocytes were also expressed in the cells obtained using our methodology. These include E11/gp38, Sost, Cox2, Mepe, Phex, and Dmp1. While Fgf23 expression was not detected in cells within digest 9 from young and old mice or the outgrowth of cells from homogenized bone particles from old mice, it was highly expressed in the total bone lysate and SW3 controls and faintly in the outgrowth of cells from homogenized young bone particles. $F g f 23$ expression is down-regulated by DMP1 and PHEX and up-regulated by MEPE, 1,25-dihydroxyvitamin $\mathrm{D}_{3}$, and PTH by unknown mechanisms $(48,49)$. In IDG-SW3 cells, Fgf 23 expression was detected after 2 weeks in culture under mineralizing conditions, therefore it appears to be a late expression gene (32). Therefore, this gene may be more quickly lost upon isolation and culture.

A limitation of our gene expression results is that they are representative of the total population of cells obtained during each stage of the procedure and not individual cells. Therefore, it remains possible that not all of the E11/GP38 expressing cells (nearly 100\% of the cells obtained during the final stages of the procedure) express each of the genes listed above. In fact, this would be expected, as the procedure should lead to the isolation of both early and late osteocytes, which are known to differ in Dmpl and Sost expression in vivo. Thus, a limitation of the procedure is that a mixed population of cells is obtained during each digest; however, this is a common problem with the use of primary cells and certainly not unique to isolating osteocytes.

This isolation technique can be used in a number of ways. By combining our osteocyte isolation procedure with fluorescence-activated cell sorting (FACS) to sort pure populations of E11/GP38+ cells from bone, a more homogeneous population could be obtained, and cells isolated from the earlier digestions could be included as well. We believe that FACS holds promise because we found that the cells did not require permeabilization for E11/GP38 staining. Whether or not E11/ GP38 staining can be achieved in unpermeablized, live cells and used to obtain a highly enriched population of osteocytes or osteocyte-like cells remains to be tested. A similar alternative would be to use this isolation procedure to obtain cells from the bones of transgenic mice carrying the green fluorescent protein (GFP) under control of the Dmp-I promoter (50) and sort Dmp-1 expressing cells (osteocytes) using FACS.

This methodology makes it possible to directly compare osteocytes obtained from young adult and aged adult bone in several important ways including age-related differ-

三HyGLO

Chemiluminescent HRP Antibody Detection Reagent

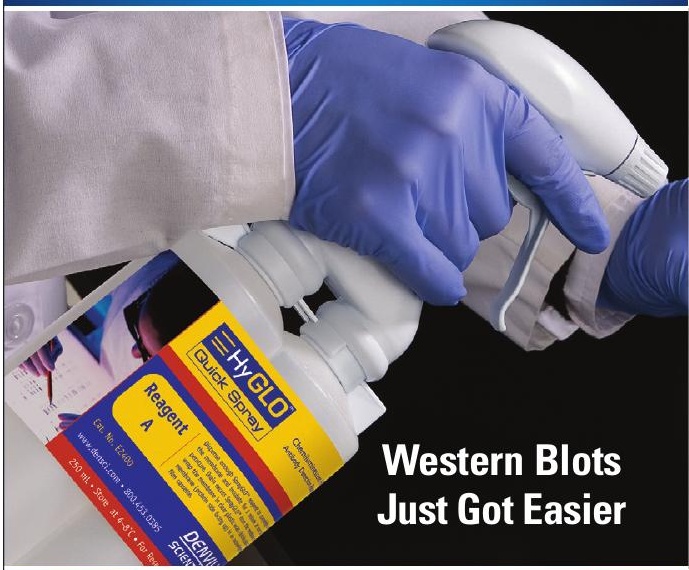

Apply ECL Reagent

\section{in a \\ Matter of Seconds}

- Simplify Your Western Blots

- Convenient, No Mixing/Measuring

- Strong Signal/Low Background

- Rapid Activation, Sensitive to 1 pg

HyGLO $^{\text {Tm }}$ Quick Spray provides instant synchronized mixing of two reagents for detection sensitivity to one picogram of antigen. The chemiluminescent signal can be detected on autoradiography film, such as $H y B$ Blot $E S^{\circledR}$.

Call for a FREE Sample Spray Bottle $\$ 39$ Value! (Cat \#E2400-SA) Supplies are limited, so act now! (One sample per lab) 
Aspirate

Fluidily

BV/G Fluid Aspiration Systems

The NEW BVC family of fluid aspiration systems deliver compact and quiet solutions for cell culture work. They provide the flexibility to accommodate your decontamination protocol whether you autoclave or disinfect in the bottle.
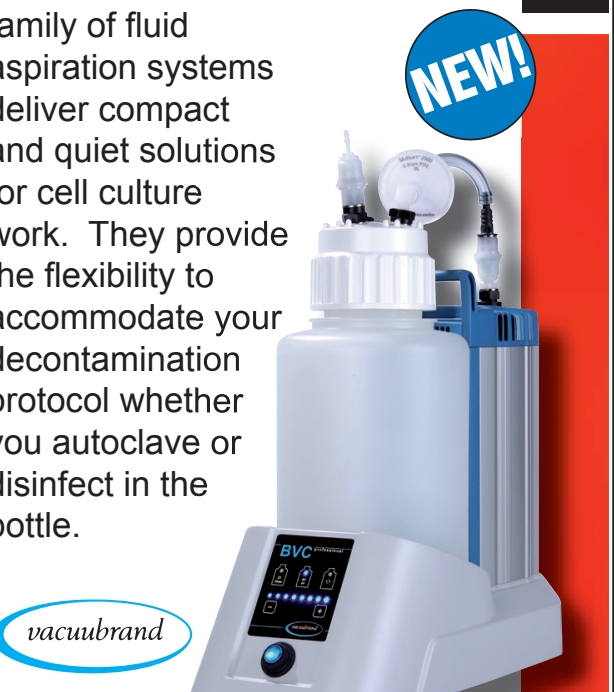

ences in mechanosensitivity that may play

- Reliable: VACUUBRAND ${ }^{\circledR}$ pump* for long service life

- Power \& Control: Suitable for two users, with adjustable* vacuum level

- Robust: "G" models designed for in-bottle bleach decontamination

- Safe: aerosol protection; models with contact-free level sensor available

\section{Culture, refined!}

${ }^{*}$ Control \& Professional models

\section{Buy from preferred dealers.}

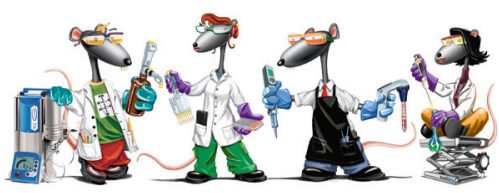

Lah Rats Trust BrandTech ${ }^{\circledR !}$

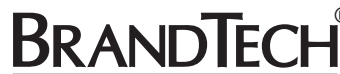
SCIENTIFIC, INC. a role in the deterioration of bone quality with age and in diseases such as osteoporosis. Osteocytes could also be obtained from mouse models of various diseases, different strains of mice with different bone characteristics, and transgenic mice designed to test specific hypotheses related to osteocyte biology. Our future work will include attempts to utilize the methodology described herein to obtain primary osteocytes from bone samples procured from both nonhuman primates and clinical bone samples. With the additional capability of isolating and culturing osteocytes procured from young and old skeletally mature mice, a new area of osteocyte research becomes available where total reliance on osteocyte-like cell lines is no longer necessary.

\section{Acknowledgments}

A portion of the funding was provided by a grant from the University of Missouri Research Board (A.R.S.) and National Institutes of Health (NIH) PO1 AR046798 (L.F.B.). The authors would also like to acknowledge Jennifer Rosser and Hong (Carrie) Zhao for their technical assistance.

\section{Competing interests}

The authors declare no competing interests.

et al. 2006. E11/gp38 selective expression in osteocytes: regulation by mechanical strain and role in dendrite elongation. Mol. Cell. Biol. 26:4539-4552.

10. You, L., S. Temiyasathit, P. Lee, C.H. Kim, P. Tummala, W. Yao, W. Kingery, A.M. Malone, et al. 2008. Osteocytes as mechanosensors in the inhibition of bone resorption due to mechanical loading. Bone 42:172-179.

11. Alford, A.I., C.R. Jacobs, and H.J. Donahue. 2003. Oscillating fluid flow regulates gap junction communication in osteocytic MLO-Y4 cells by an ERK1/2 MAP kinase-dependent mechanism small star, filled. Bone 33:64-70.

12. Genetos, D.C., C.J. Kephart, Y. Zhang, C.E. Yellowley, and H.J. Donahue. 2007. Oscillating fluid flow activation of gap junction hemichannels induces ATP release from MLO-Y4 osteocytes. J. Cell. Physiol. 212:207-214.

13. Himpens, B. and J. Vereecke. 2000. Intra- and intercellular $\mathrm{Ca}(2+)$-signal transduction. Verh. K. Acad. Geneeskd. Belg. 62:501-563.

14. Cheng, B., S. Zhao, J. Luo, E. Sprague, L.F. Bonewald, and J.X. Jiang. 2001. Expression of functional gap junctions and regulation by fluid flow in osteocyte-like MLO-Y4 cells. J. Bone Miner. Res. 16:249-259.

15. Cherian, P.P., A.J. Siller-Jackson, S. Gu, X. Wang, L.F. Bonewald, E. Sprague, and J.X. Jiang. 2005. Mechanical strain opens connexin 43 hemichannels in osteocytes: a novel mechanism for the release of prostaglandin. Mol. Biol. Cell 16:3100-3106.

16. Ponik, S.M., J.W. Triplett, and F.M. Pavalko. 2007. Osteoblasts and osteocytes respond differently to oscillatory and unidirectional fluid flow profiles. J. Cell. Biochem. 100:794-807.

17. Gross, T.S., K.A. King, N.A. Rabaia, P. Pathare, and S. Srinivasan. 2005. Upregulation of osteopontin by osteocytes deprived of mechanical loading or oxygen. J. Bone Miner. Res. 20:250256.

\section{References}

1. Bonewald, L.F. 2007. Osteocytes as dynamic multifunctional cells. Ann. N. Y. Acad. Sci. 1116:281290.

2. Bonewald, L.F. 2007. Osteocyte messages from a bony tomb. Cell Metab. 5:410-411.

3. Bonewald, L.F. and M.L. Johnson. 2008. Osteocytes, mechanosensing and Wnt signaling. Bone 42:606-615.

4. Bonewald, L.F. 1999. Establishment and characterization of an osteocyte-like cell line, MLO-Y4. J. Bone Miner. Metab. 17:61-65.

5. Kato, Y., J.J. Windle, B.A. Koop, G.R. Mundy, and L.F. Bonewald. 1997. Establishment of an osteocyte-like cell line, MLO-Y4. J. Bone Miner. Res. 12:2014-2023.

6. Yang, W., M.A. Harris, J.G. Heinrich, D. Guo, L.F. Bonewald, and S.E. Harris. 2009. Gene expression signatures of a fibroblastoid preosteoblast and cuboidal osteoblast cell model compared to the MLO-Y4 osteocyte cell model. Bone 44:32-45.

7. Cheng, B., Y. Kato, S. Zhao, J. Luo, E. Sprague, L.F. Bonewald, and J.X. Jiang. 2001. PGE(2) is essential for gap junction-mediated intercellular communication between osteocyte-like MLO-Y4 cells in response to mechanical strain. Endocrinology 142:3464-3473.

8. Cherian, P.P., B. Cheng, S. Gu, E. Sprague, L.F. Bonewald, and J.X. Jiang. 2003. Effects of mechanical strain on the function of Gap junctions in osteocytes are mediated through the prostaglandin EP2 receptor. J. Biol. Chem. 278:43146-43156.

9. Zhang, K., C. Barragan-Adjemian, L. Ye, S. Kotha, M. Dallas, Y. Lu, S. Zhao, M. Harris,
18. Reilly, G.C., T.R. Haut, C.E. Yellowley, H.J. Donahue, and C.R. Jacobs. 2003. Fluid flow induced PGE2 release by bone cells is reduced by glycocalyx degradation whereas calcium signals are not. Biorheology 40:591-603.

19. Vatsa, A., D. Mizuno, T.H. Smit, C.F. Schmidt, F.C. MacKintosh, and J. Klein-Nulend. 2006. Biol. imaging of intracellular NO production in single bone cells after mechanical stimulation. J. Bone Miner. Res. 21:1722-1728.

20. Kurata, K., T.J. Heino, H. Higaki, and H.K. Vaananen. 2006. Bone marrow cell differentiation induced by mechanically damaged osteocytes in 3D gel-embedded culture. J. Bone Miner. Res. 21:616-625.

21. Robling, A.G., P.J. Niziolek, L.A. Baldridge, K.W. Condon, M.R. Allen, I. Alam, S.M. Mantila, J. Gluhak-Heinrich, et al. 2008. Mechanical stimulation of bone in vivo reduces osteocyte expression of Sost/sclerostin. J. Biol. Chem. 283:5866-5875.

22. Winkler, D.G., M.K.Sutherland,J.C. Geoghegan, C. Yu, T. Hayes, J.E. Skonier, D. Shpektor, M. Jonas, et al. 2003. Osteocyte control of bone formation via sclerostin, a novel BMP antagonist. EMBO J. 22:6267-6276.

23. Bellido, T. 2006. Summary - Osteocyte control of bone formation via Sost/sclerostin. J. Musculoskelet. Neuronal Interact. 6:360-363.

24. Harris, S.E., J. Gluhak-Heinrich, M.A. Harris, W. Yang, L.F. Bonewald, D. Riha, P.S. Rowe, A.G. Robling, et al. 2007. DMP1 and MEPE expression are elevated in osteocytes after mechanicalloading 
in vivo: theoretical role in controlling mineral quality in the perilacunar matrix. J. Musculoskelet. Neuronal Interact. 7:313-315.

25. Lu, Y., Y. Xie, S. Zhang, V. Dusevich, L.F. Bonewald, and J.Q. Feng. 2007. DMP1-targeted Cre expression in odontoblasts and osteocytes. J. Dent. Res. 86:320-325.

26. Rios, H.F., L. Ye, V. Dusevich, D. Eick, L.F. Bonewald, and J.Q. Feng. 2005. DMP1 is essential for osteocyte formation and function. J. Musculoskelet. Neuronal Interact. 5:325-327.

27. Toyosawa, S., S. Shintani, T. Fujiwara, T. Ooshima, A. Sato, N. Ijuhin, and T. Komori. 2001. Dentin matrix protein 1 is predominantly expressed in chicken and rat osteocytes but not in osteoblasts. J. Bone Miner. Res. 16:2017-2026.

28. Gluhak-Heinrich, J., L. Ye, L.F. Bonewald, J.Q. Feng, M. MacDougall, S.E. Harris, and D. Pavlin. 2003. Mechanical loading stimulates dentin matrix protein 1 (DMP1) expression in osteocytes in vivo. J. Bone Miner. Res. 18:807-817.

29. Yang, W., Y. Lu, I. Kalajzic, D. Guo, M.A. Harris, J. Gluhak-Heinrich, S. Kotha, L.F. Bonewald, et al. 2005. Dentin matrix protein 1 gene cis-regulation: use in osteocytes to characterize local responses to mechanical loading in vitro and in vivo. J. Biol. Chem. 280:20680-20690.

30.Kato, Y., A. Boskey, L. Spevak, M. Dallas, M. Hori, and L.F. Bonewald. 2001. Establishment of an osteoid preosteocyte-like cell MLO-A5 that spontaneously mineralizes in culture. J. Bone Miner. Res. 16:1622-1633.

31. Barragan-Adjemian, C., D. Nicolella, V. Dusevich, M.R. Dallas, J.D. Eick, and L.F. Bonewald. 2006. Mechanism by which MLO-A5 late osteoblasts/ early osteocytes mineralize in culture: similarities with mineralization of lamellar bone. Calcif. Tissue Int. 79:340-353

32.Woo, S.M., J. Rosser, V. Dusevich, I. Kalajzic, and L.F. Bonewald. 2011. Cell line IDG-SW3 replicates osteoblast-to-late-osteocyte differentiation in vitro and accelerates bone formation in vivo. $\mathrm{J}$. Bone Miner. Res. 26:2634-2646.

33. Gu, G., M. Nars, T.A. Hentunen, K. Metsikko, and H.K. Vaananen. 2006. Isolated primary osteocytes express functional gap junctions in vitro. Cell Tissue Res. 323:263-271.

34. Gu, G., T.A. Hentunen, M. Nars, P.L. Harkonen, and H.K. Vaananen. 2005. Estrogen protects primary osteocytes against glucocorticoidinduced apoptosis. Apoptosis 10:583-595.

35. Murshid, S.A., H. Kamioka, Y. Ishihara, R. Ando, Y. Sugawara, and T. Takano-Yamamoto. 2007. Actin and microtubule cytoskeletons of the processes of 3D-cultured MC3T3-E1 cells and osteocytes. J. Bone Miner. Metab. 25:151-158.

36.Nijweide, P.J., A. Van der Plas, M.J. Alblas, and J. klein-Nulend. 2003. Osteocyte Isolation and Culture, p. 41-50. In M.H. Helfrich, and S. Ralston (Eds.), Methods in Molecular Medicine: Bone Research Protocols. Humana Press, Totowa, NJ.

37. Tanaka-Kamioka, K., H. Kamioka, H. Ris, and S.S. Lim. 1998. Osteocyte shape is dependent on actin filaments and osteocyte processes are unique actin-rich projections. J. Bone Miner. Res. 13:1555-1568

38. Kamioka, H., T. Honjo, and T. TakanoYamamoto. 2001. A three-dimensional distribution of osteocyte processes revealed by the combination of confocal laser scanning microscopy and differential interference contrast microscopy. Bone 28:145-149.

39. Kamioka, H., Y. Ishihara, H. Ris, S.A. Murshid, Y. Sugawara, T. Takano-Yamamoto, and S.S. Lim. 2007. Primary cultures of chick osteocytes retain functional gap junctions between osteocytes and between osteocytes and osteoblasts. Microsc. Microanal. 13:108-117.

40. Kamioka, H., Y. Miki, K. Sumitani, K. Tagami, K. Terai, K. Hosoi, and T. Kawata. 1995. Extracellular calcium causes the release of calcium from intracellular stores in chick osteocytes. Biochem. Biophys. Res. Commun. 212:692-696.

41. Kamioka, H., Y. Sugawara, S.A. Murshid, Y Ishihara, T. Honjo, and T. Takano-Yamamoto. 2006. Fluid shear stress induces less calcium response in a single primary osteocyte than in a single osteoblast: implication of different focal adhesion formation. J. Bone Miner. Res. 21:10121021

42. Pitsillides, A.A., S.C. Rawlinson, R.F. Suswillo, S. Bourrin, G. Zaman, and L.E. Lanyon. 1995. Mechanical strain-induced NO production by bone cells: a possible role in adaptive bone (re) modeling? FASEB J. 9:1614-1622.

43. Westbroek, I., N.E. Ajubi, M.J. Alblas, C.M. Semeins, J. Klein-Nulend, E.H. Burger, and P.J. Nijweide. 2000. Differential stimulation of prostaglandin $\mathrm{G} / \mathrm{H}$ synthase-2 in osteocytes and other osteogenic cells by pulsating fluid flow. Biochem. Biophys. Res. Commun. 268:414-419.

44. van der Plas, A. and P.J. Nijweide. 1992. Isolation and purification of osteocytes. J. Bone Miner. Res. 7:389-396

45. Xiao, Z., S. Zhang, J. Mahlios, G. Zhou, B.S. Magenheimer, D. Guo, S.L. Dallas, R. Maser, et al. 2006. Cilia-like structures and polycystin-1 in osteoblasts/osteocytes and associated abnormalities in skeletogenesis and Runx 2 expression. J. Biol. Chem. 281:30884-30895.

46.Zhao, S., Y.K. Zhang, S. Harris, S.S. Ahuja, and L.F. Bonewald. 2002. MLO-Y4 osteocyte-like cells support osteoclast formation and activation. J. Bone Miner. Res. 17:2068-2079.

47. van der Plas, A. and P.J. Nijweide. 2005. JBMR anniversary classic. Isolation and purification of osteocytes. A van der Plas A, PJ Nijweide. Originally published in Volume 7, Number 4, pp 389-96 (1992). J. Bone Miner. Res. 20:706-714

48. Lavi-Moshayoff, V., G. Wasserman, T. Meir, J. Silver, and T. Naveh-Many. 2010. PTH increases FGF23 gene expression and mediates the high-FGF23 levels of experimental kidney failure: a bone parathyroid feedback loop. Am. J. Physiol. Renal Physiol. 299:F882-F889.

49.Martin, A., S. Liu, V. David, H. Li, A. Karydis, J.Q. Feng, and L.D. Quarles. 2011. Bone proteins PHEX and DMP1 regulate fibroblastic growth factor Fgf23 expression in osteocytes through a common pathway involving FGF receptor (FGFR) signaling. FASEB J. 25:2551-2562.

50. Kalajzic, I., A. Braut, D. Guo, X. Jiang, M.S. Kronenberg, M. Mina, M.A. Harris, S.E. Harris, and D.W. Rowe. 2004. Dentin matrix protein 1 expression during osteoblastic differentiation, generation of an osteocyte GFP-transgene. Bone 35:74-82.

Received 4 November 2011; accepted 18 May 2012.

Address correspondence to Amber Rath Stern, Mechanical Engineering and Oral Biology, University of Missouri-Kansas City, 370G Robert H. Flarsheim Hall, 5100 Rockhill Road, Kansas City, MO, USA.Email: sterna@umkc.edu

To purchase reprints of this article, contact: biotechniques@fosterprinting.com
Meet our new family...

क

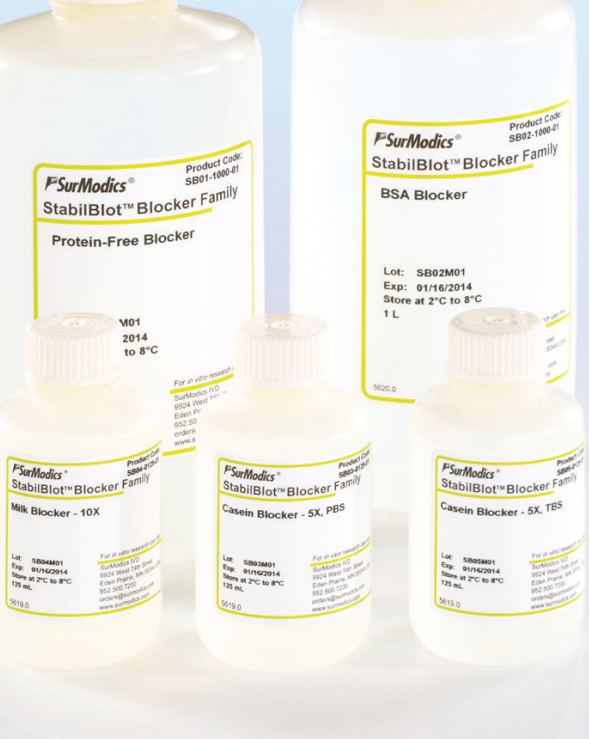

StabilBlot ${ }^{\mathrm{TM}}$ Blocker Family

- Protein Stabilizers / Blockers

- BioFX ${ }^{\circledR}$ Substrates / Stop Reagents

- Diluents / Buffers / IHC Reagents / Accessory Reagents

- DIARECT ${ }^{\mathrm{TM}}$ Autoimmune / Infectious Disease Antigens

- BioFX ${ }^{\circledR}$ Antibodies / Streptavidin

- Microarray Slides / Surfaces

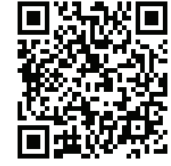

\section{Visit us at \\ ASM and AACC}

\section{FSurModics}

orders@surmodics.com

952-500-7200

www.surmodics.com 